$\xi=-1$

\title{
Design of Memristive Hopfield Neural Network using Memristor Bridges
}

\author{
Monurajan $\mathbf{P}^{1}$, Ruhanbevi $\mathbf{A}^{2}$, Manjula $\mathbf{J}^{3}$ \\ ${ }^{1,2,3}$ Department of Electronics and Comunication, \\ SRM Institute of Science and Technology, SRM Nagar, Kattankulathur, India \\ *Corresponding Author Email: ${ }^{1}$ monurajan_rajanpeter@srmuniv.edu.in, ${ }^{2}$ ruhan.b@ktr.srmuniv.ac.in \\ ${ }^{3}$ manjula.j@ktr.srmuniv.ac.in
}

\begin{abstract}
Artificial Neural Networks are interconnection of neurons inspired from the biological neural network of the brain. ANN is claimed to rule the future, spreads its wings to various areas of interest to name a few such as optimization, information technology, cryptography, image processing and even in medical diagnosis. There are devices which possess synaptic behaviour, one such device is memristor. Bridge circuit of memristors can be combined together to form neurons. Neurons can be made into a network with appropriate parameters to store data or images. Hopfield neural networks are chosen to store the data in associative memory. Hopfield neural networks are a significant feature in ANN which are recurrent in nature and in general are used as associative memory and in solving optimization problems such as the Travelling Salesman Problem. The paper deals on the construction of memristive Hopfield neural network using memristor bridging circuit and its application in the associative memory. This paper also illustrates the experiment with mathematical equations and the associative memory concept of the network using Matlab.
\end{abstract}

Keywords: Bridge circuit, Hopfield neural network, Memristor.

\section{Introduction}

The digital World is facing lot of problems with data security. The interchanging of the data demands to have features such as secure, reliable and persistent. Artificial Neural Networks (ANN) provides efficient cryptographic application. ANN are inspired from the biological human brain network [1]. For example, the Hopfield memory can associate data or images similar to that of a human brain. ANN has diff erent architectures such as the single layer, multi-layer and recurrent networks. In order to secure data, the data must be stored in the memory. There is been an urge to develop machines which can have associative memory that of humans. Hopfield networks tends to be one that has the ability to associate with data types.

Experiments on the Hopfield networks for binary image convergence [2] was done to prove the point. Hopfield networks can even be used for solving TSP, optimization and other crucial problems. The introduction of memristors as the fourth basic element by Chua [3] and the demonstration of existing of the new nanoscale electronic component by Strukov et al. [4] triggered interest in the people. Many scholars proposed that memristors possess synaptic behaviour. The memristance of the memristor were used to calculate the synaptic weights in some cases. In 2015, Wang et al. [5] Pavlov experiments showed relations between the memristors and the associative memory, also with the MHN [6] single and multi associative memories have been realised. Tarkov [7] in 2016 conducted experiments on the binary image convergence in associative memory.
The implementation of the experiment was done with Hopfield associative memory with interneuronal connections through bridges using memristors.

The paper deals with the construction of neuron using synapse of memristor bridge circuits. Matrix Laboratory (MATLAB) software is to be used for the processing and simulations of the results.

A minimum of three neurons are taken into account for the generation of three bits data. Hopfield neural network is chosen for the network operations to be carried out. The Memristive Hopfield neural network acts as an associative memory unit.

\section{Proposed Memristor Bridge Circuit and Neuron Model}

Memristors possess current and magnetic flux linkages. The relationship between voltage and current of a memristor is given by

$\mathrm{v}=(\operatorname{Ron}(\mathrm{w} / \mathrm{D}))+(\operatorname{Roff}(1(\mathrm{w} / \mathrm{D}))) \mathrm{i}$

In the above equation, $\mathrm{D}$ is the device length in $\mathrm{nm}$ Ron is the on (minimum) resistance of the device Roff is the of (maximum) resistance of the device $\mathrm{w}$ is the weight from the memristor $\mathrm{v}$ is the voltage and $i$ is the current. Four of the memristors are combined together to form bridge circuit. Two of the memristors should be connected in forward bias and the other two memristors in the reverse bias. The input voltage and ground is given between the forward and the reverse bias of the circuit. The output voltage is received at the junction between the similarly biased memristors. A non-inverting negative feedback operational amplifier is used to find the potential diff erence between the junction points $\mathrm{A}$ and $\mathrm{B}$ as shown in Figure 1 Voltages across the memristors in the memristor bridge are given by, 


$$
\begin{aligned}
& \mathrm{VM} 1=(\mathrm{M} 1(\mathrm{M} 1+\mathrm{M} 2)) \cdot \mathrm{Vin} \\
& \mathrm{VM} 2=(\mathrm{M} 2(\mathrm{M} 1+\mathrm{M} 2)) \cdot \mathrm{Vin}=\mathrm{VA} \\
& \mathrm{VM} 3=(\mathrm{M} 3(\mathrm{M} 3+\mathrm{M} 4)) \cdot \mathrm{Vin} \\
& \mathrm{VM} 4=(\mathrm{M} 4(\mathrm{M} 3+\mathrm{M} 4)) \cdot \mathrm{Vin}=\mathrm{VB}
\end{aligned}
$$

Here, M1, M2, M3, M4 are the memristances of the memristors; $\mathrm{VM} 1, \mathrm{VM} 2, \mathrm{VM} 3, \mathrm{VM} 4$ are the voltages across the memristors and VA, VB are the voltages at the junctions A and B respectively.

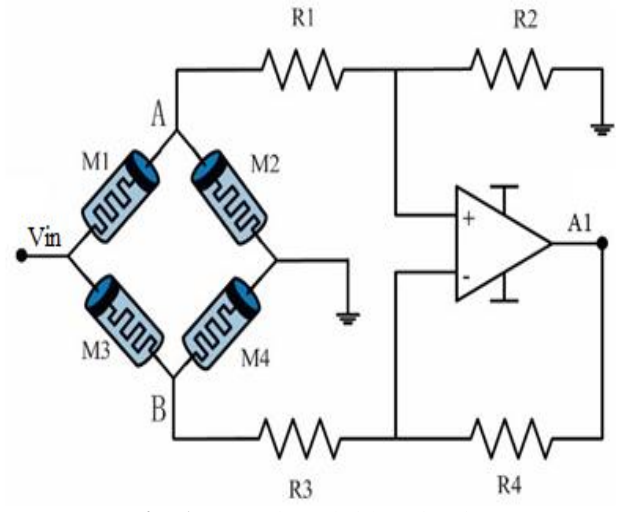

Fig. 1: Memristor bridge circuit

The output voltage or the voltage between $\mathrm{A}$ and $\mathrm{B}$ terminal is by

$$
\mathrm{A} 1=\mathrm{VA}-\mathrm{VB}
$$

The synaptic weight of the memristor bridge is given by

$$
\psi=(\mathrm{M} 2(\mathrm{M} 1+\mathrm{M} 2))-(\mathrm{M} 4(\mathrm{M} 3+\mathrm{M} 4))
$$

The output voltage of the circuit is given by the product of the synaptic weight and the input voltage as follows

$$
\mathrm{A} 1=((\mathrm{M} 2(\mathrm{M} 1+\mathrm{M} 2))-(\mathrm{M} 4(\mathrm{M} 3+\mathrm{M} 4))) . \mathrm{Vin}
$$

(or)

$$
\mathrm{A} 1=\psi * \mathrm{Vin}
$$

With the adjustments in the memristive values the synaptic weights of the bridge circuit can be controlled to be positive, negative or to be at zero. If $\mathrm{M} 2 / \mathrm{M} 1>\mathrm{M} 4 / \mathrm{M} 3$ then the synaptic weight will be positive. Similarly if M2/M1 <M4/M3 the synaptic weight will be negative and $\mathrm{M} 2 / \mathrm{M} 1=\mathrm{M} 4 / \mathrm{M} 3$ will account to a zero synaptic weight.

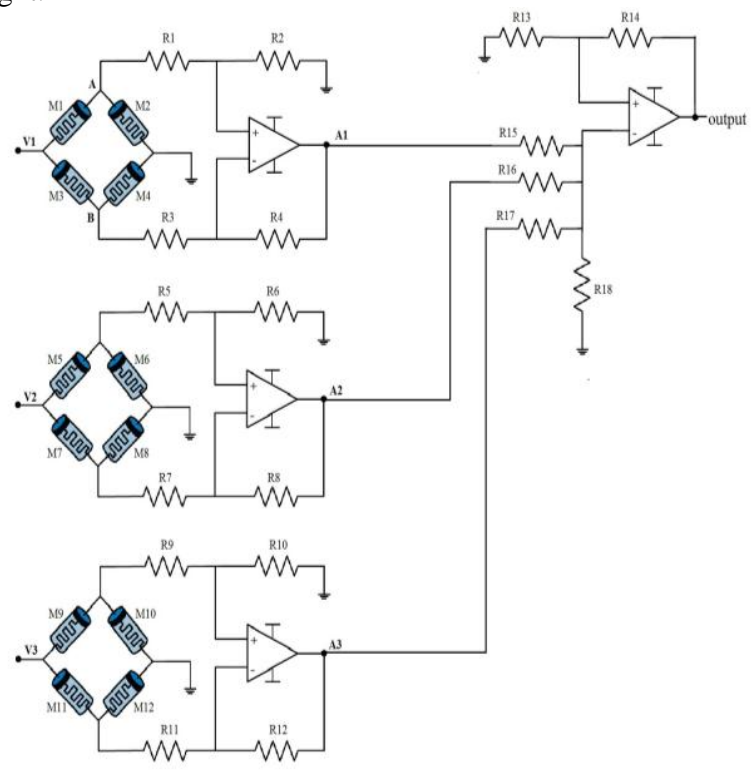

Fig. 2: Proposed Neuron Mode
Each of the memristor bridge circuit possess synaptic behaviour. Three of the synapse producing bridge circuits which can be controlled by the input voltages are to be combined. The complete neuron model consists of three memristor bridge circuits combined together with a non-inverting negative feedback amplifier as shown in Figure 2. The output of the neuron depends upon the synapticweight, input and the threshold factor, which means there will a specified threshold (say, T) in addition to the output of the memristor bridges. The output of the neuron is given by

$$
\sum_{j=1}^{3}\left(A_{j}\right)-T
$$

The output of the neurons are the combined sum of the synaptic weights of the memristor bridge circuits connected to it along with some threshold value.

\section{Proposed Memristive Hopfield Neural Network}

In the Hopfield neural network, each of the neurons are given to other neurons. Self connection of neurons are not done. The weight of the network follows a $3 \times 3$ matrix of the following

$\left[\begin{array}{lll}W 11 & W 12 & W 13 \\ W 21 & W 22 & \text { W23 } \\ \text { W31 } & \text { W32 } & \text { W33 }\end{array}\right]$

The weights of the network are symmetric and the elements in the diagonal matrix are considered to be zero since no self connection is possible. The symmetricity of the weight matrix means that $\mathrm{W} 12=\mathrm{W} 21, \mathrm{~W} 13=\mathrm{W} 31$, and $\mathrm{W} 23=\mathrm{W} 32$.

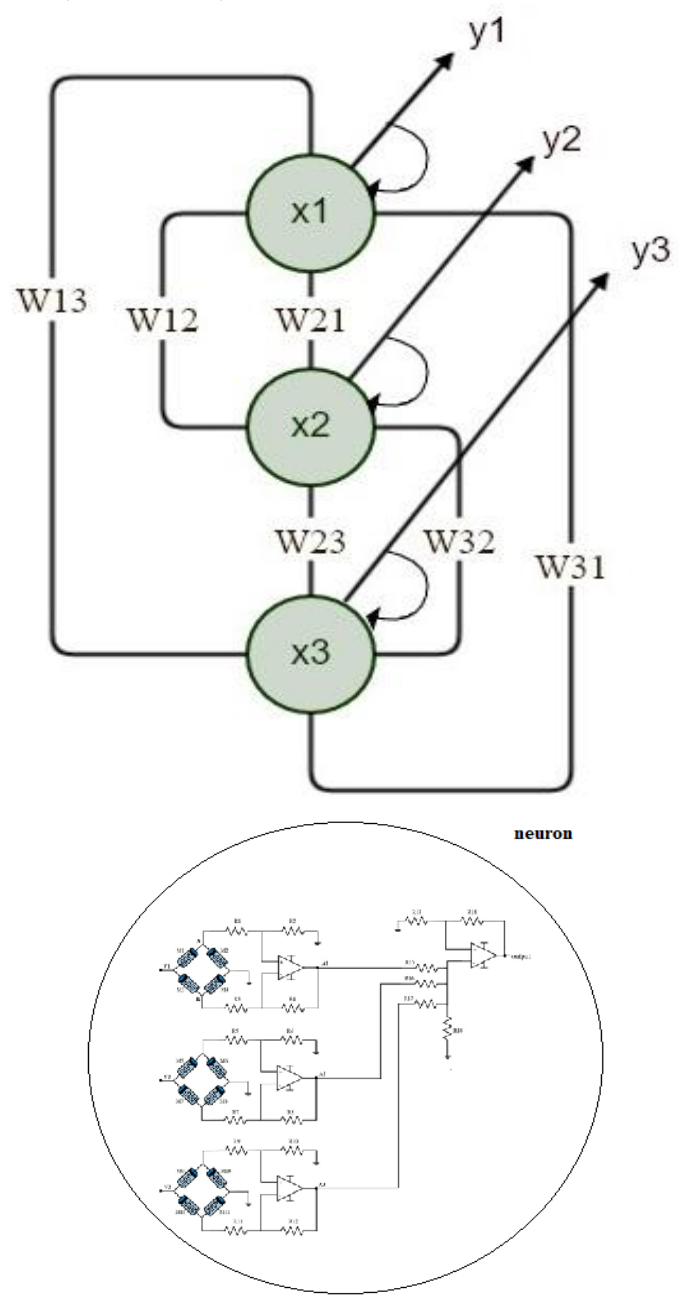

Fig. 3: Proposed memristive Hopfield neural network 
The Hopfield neural network in which the neurons are constructed with the involvement of the memristors are termed as memristive Hopfield neural network. The proposed work can be illustrated using a state diagram as in Figure 3 with $\mathrm{x} 1$, x2, x3 neurons, W12, $\mathrm{W} 13, \mathrm{~W} 21, \mathrm{~W} 23, \mathrm{~W} 31, \mathrm{~W} 32$ as the weights and $\mathrm{y} 1, \mathrm{y} 2, \mathrm{y} 3$ as the output of the neural network. The neurons in the network are considered to be the neuron model constructed from the memristor bridge circuit. The output of the neurons are updated one by one in a sequential fashion. The output of each of the neuron is given as input to the next neuron model.

\section{Proposed Associative Memory and Output}

\section{A. Single Associative Memory}

In single associative memory all the eight sets of three bit pattern are associated to a single set of three bit pattern, which is stored in the associative memory. The pattern to be stored is fixed and according to the pattern which has to be stored, the weights are to be devised. The memristance in the memristor bridge circuits are chosen according to the weights and the input are given by adjusting the corresponding input voltages to the memristor bridge circuits. Inorder to design a model with memristance values such that the required pattern of three bit data can be storeda set of weights are calculated. According to the weights the memristance values are then set such that the required pattern of three bit data is associated. The weights, so as to store the three bit data pattern of 101 is given by

$1 / 50\left[\begin{array}{ccc}0 & -2.6 & 2 \\ -2.6 & 0 & 0.92 \\ 2 & 0.92 & 0\end{array}\right]$

The memristance values for the corresponding weights of the stored pattern can be given by

$\mathrm{M} 1=\mathrm{M} 17=\mathrm{M} 33=\mathrm{M} 3=\mathrm{M} 19=\mathrm{M} 35=8$;

$\mathrm{M} 2=\mathrm{M} 18=\mathrm{M} 34=\mathrm{M} 4=\mathrm{M} 20=\mathrm{M} 36=10$;

M5 $=$ M13 $=8.359 ;$ M6= M14=9.4;

$\mathrm{M} 7=\mathrm{M} 15=7.54 ; \mathrm{M} 8=\mathrm{M} 16=10.47$;

$\mathrm{M} 9=\mathrm{M} 25=7.64 ; \mathrm{M} 10=\mathrm{M} 26=10.36$;

$\mathrm{M} 11=\mathrm{M} 27=8.36 ; \mathrm{M} 12=\mathrm{M} 28=9.64$;

$\mathrm{M} 21=\mathrm{M} 29=7.83 ; \mathrm{M} 22=\mathrm{M} 30=10.16$;

$\mathrm{M} 23=\mathrm{M} 31=8.17 ; \mathrm{M} 24=\mathrm{M} 32=9.84$

For each of the input 3 bit pattern the 3 bit data patterns are associated with the same bit pattern of 101. Each bit of the input bit pattern update in the memristive Hopfield network inorder to attain at the estimated output pattern. The output with the updation pattern (input $\rightarrow$ updation states $\rightarrow$ output) can be given by

$000 \rightarrow 100 \rightarrow 100 \rightarrow 101$

$001 \rightarrow 101 \rightarrow 101 \rightarrow 101$

$010 \rightarrow 110 \rightarrow 100 \rightarrow 101$

$100 \rightarrow 100 \rightarrow 100 \rightarrow 101$

$101 \rightarrow 101 \rightarrow 101 \rightarrow 101$

$110 \rightarrow 110 \rightarrow 100 \rightarrow 101$

$111 \rightarrow 111 \rightarrow 101 \rightarrow 101$

If the same pattern is inputed the output will be same since the updation of pattern will result in the output pattern which is equal to the input pattern.

\section{B. Multi Associative Memory}

Hopfield network can be devised for the multi associative memory. In multi association memory for the given set of data input bits multiple number bits can be associated. Similar to that of the single associative memory the weights for the patterns 101 and 000 can be given by

$1 / 50\left[\begin{array}{ccc}0 & 0.5 & 1.5 \\ 0.5 & 0 & 0.25 \\ 1.5 & 0.25 & 0\end{array}\right]$

The corresponding memristance values for the weights can be given as follows,

$\mathrm{M} 1=\mathrm{M} 17=\mathrm{M} 33=\mathrm{M} 3=\mathrm{M} 19=\mathrm{M} 35=8 ;$

$\mathrm{M} 2=\mathrm{M} 18=\mathrm{M} 34=\mathrm{M} 4=\mathrm{M} 20=\mathrm{M} 36=10$;

$\mathrm{M} 5=\mathrm{M} 13=7.91 ; \mathrm{M} 6=\mathrm{M} 14=10.09$;

$\mathrm{M} 7=\mathrm{M} 15=8.09 ; \mathrm{M} 8=\mathrm{M} 16=9.91$;

$\mathrm{M} 9=\mathrm{M} 25=7.73 ; \mathrm{M} 10=\mathrm{M} 26=10.27$

$\mathrm{M} 11=\mathrm{M} 27=8.27 ; \mathrm{M} 12=\mathrm{M} 28=9.73$;

$\mathrm{M} 21=\mathrm{M} 29=7.95 ; \mathrm{M} 22=\mathrm{M} 30=10$;

$\mathrm{M} 23=\mathrm{M} 31=8.1 ; \mathrm{M} 24=\mathrm{M} 32=9.985$

Similar to the single associative memory the output bits are updated bit by bit. The output and the updation pattern of the bits(input $\rightarrow$ updation states $\rightarrow$ output) can be given by

$000 \rightarrow 000 \rightarrow 000 \rightarrow 000$
$001 \rightarrow 101 \rightarrow 101 \rightarrow 101$
$010 \rightarrow 010 \rightarrow 000 \rightarrow 000$
$100 \rightarrow 000 \rightarrow 000 \rightarrow 001$
$101 \rightarrow 101 \rightarrow 101 \rightarrow 101$
$110 \rightarrow 010 \rightarrow 000 \rightarrow 000$
$111 \rightarrow 111 \rightarrow 101 \rightarrow 101$

If the memristance values are change then the corresponding weights will also change which will aff ect the association of the pattern. So it is necessary to keep the memristance values a constant eventually the synaptic weights will be constant.

\section{Conclusion}

The proposed methodology contributes for the hardware model of the association models in real time use. With the increased profound research of the memristors using $\mathrm{TiO} 2$, the availability and usage of memristors in general applications can be made possible in the nearest future. This could reduce the size and greatly improve the performance of the neural networks. The proposed method can be extended to associate for images and other greater association of the pattern in a larger scale in the image recognition and construction of images and patterns from damaged images and patterns. The synaptic behaviour of the memristive bridges contribute to the efficient functioning the entire memristive Hopfield neural network. The neuron construction using the synapses are constructed in the view of the formation of the Hopfield neural network of the proposed system. The use of the bridge circuit in other hardware and neural application are set at the future enhancement of the work.

\section{References}

[1] B. Yegnanarayana, "Artificial Neural Networks", PHI Learning ISBN9788120312531.URLhttps://books.google.co.in/books?id=RT tvUVU_xL4C, 2009.

[2] Lidan Wang, Xiaodong Wang, Shukai Duan, and Huifang Li, "A spintronic memristor bridge synapse circuit and the application in memrisitive cellular automata", Neurocomputing, 167(Supplement C):346 - 351, ISSN 0925-2312. doi: https://doi.org/10.1016/j.neucom.2015. 04.061, 2015.

[3] L. Chua. Memristor-the missing circuit element. IEEE Transactions on Circuit Theory, 18(5):507-519, ISSN 0018-9324. doi 10.1109/TCT.1971.1083337, September 1971.

[4] Dmitri B. Strukov, Gregory S. Snider, Duncan R. Stewart, and R. Stanley Williams. The missing memristor found. 453: $80 \mathrm{EP}$, URL http://dx.doi.org/10.1038/ nature06932, May 2008.

[5] Lidan Wang, Huifang Li, Shukai Duan, Tingwen Huang, and Huamin Wang. Pavlov associative memory in a memristive neura network and its circuit implementation. Neurocomputing, 171(Supplement C):23 - 29, ISSN 0925-2312. doi https://doi.org/10.1016/j.neucom.2015. 05.078, 2016. 
[6] S. G. Hu, Y. Liu, Z. Liu, T. P. Chen, J. J. Wang, Q. Yu, L. J. Deng, Y. Yin, and Sumio Hosaka. Associative memory realized by a reconfigurable memristive hopfield neural network. 6: 7522 EP Jun 2015. URL http://dx.doi.org/10.1038/ ncomms8522. Article.

[7] Mikhail S. Tarkov, "Hopfield Network with Interneuronal Connections Based on Memristor Bridges, pages 196-203. Springer International Publishing, Cham, ISBN 978-3-319-406633. doi: 10.1007/978-3-319-40663-3 23, 2016.

[8] Hyongsuk Kim, Maheshwar P. Sah, Changju Yang, Tam'as Roska, and Leon O. Chua. "Memristor Bridge-Based Artificial Neural Weighting Circuit", Springer International Publishing, Cham, ISBN 978-3-319-02630-5. doi: 10.1007/978-3-319-02630-5 12. pages 249-265. 2014.

[9] Shukai Duan, Yi Zhang, Xiaofang Hu, Lidan Wang, and Chuandong Li. "Memristor-based chaotic neural networks for associative memory", Neural Computing and Applications, 25(6):1437-1445, ISSN 1433-3058. doi: 10. 1007/s00521-014 1633-x, Nov 2014

[10] Beiye Liu, Yiran Chen, Bryant Wysocki, and Tingwen Huang. "Reconfigurable neuromorphic computing system with memristorbased synapse design", Neural Processing Letters, 41(2):159-167, ISSN 1573-773X. doi: 10. 1007/s11063-013-9315-8, Apr 2015.

[11] S. G. Hu, Y. Liu, Z. Liu, T. P. Chen, J. J. Wang, Q. Yu, L. J Deng, Y. Yin, and Sumio Hosaka, "A memristive hopfield network for associative memory", URL http://dx.doi.org/10.1038/protex.2015.070, July 2015. 